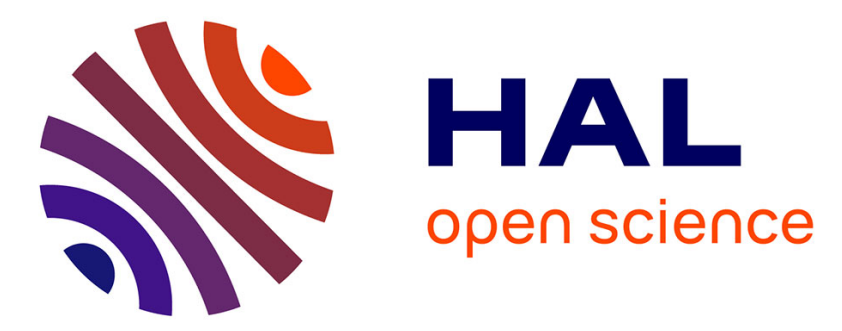

\title{
Versatile Multiview Layered Video Based on Distributed Source Coding
}

\author{
Giovanni Petrazzuoli, C. Macovei, I.-E. Nicolae, Marco Cagnazzo, Frederic \\ Dufaux, Beatrice Pesquet-Popescu
}

\section{- To cite this version:}

Giovanni Petrazzuoli, C. Macovei, I.-E. Nicolae, Marco Cagnazzo, Frederic Dufaux, et al.. Versatile Multiview Layered Video Based on Distributed Source Coding. Int. Workshop on Image and Audio Analysis for Multimedia Interactive Services (WIAMIS'2013), Jul 2013, Paris, France. hal-01436236

\section{HAL Id: hal-01436236 \\ https://hal.science/hal-01436236}

Submitted on 10 Jan 2020

HAL is a multi-disciplinary open access archive for the deposit and dissemination of scientific research documents, whether they are published or not. The documents may come from teaching and research institutions in France or abroad, or from public or private research centers.
L'archive ouverte pluridisciplinaire HAL, est destinée au dépôt et à la diffusion de documents scientifiques de niveau recherche, publiés ou non, émanant des établissements d'enseignement et de recherche français ou étrangers, des laboratoires publics ou privés. 


\title{
VERSATILE MULTIVIEW LAYERED VIDEO BASED ON DISTRIBUTED SOURCE CODING
}

\author{
G. Petrazzuoli, C. Macovei, I.-E. Nicolae, M. Cagnazzo, F. Dufaux, B. Pesquet-Popescu \\ Institut Mines-Télécom, Télécom-ParisTech / CNRS LTCI / UMR 5141
}

\begin{abstract}
Video content constitutes today a large part of the data traffic on the Internet. This is allowed by the capillary spreading of video codec technologies: nowadays, every computer, tablet and smart phone is equipped with video encoding and decoding technologies. As a matter of fact, the video content often exists in different formats, that, even though they can be incompatible to each other, still have a significant mutual redundancy. The incompatibility prevents an efficient exploitation of the scalability, which on the other hand is a very important characteristic when it comes to efficient network use.

An interesting alternative to classical scalable video is to use distributed video coding (DVC) for the enhancement layers. In the envisaged scenario, clients have different decoders for the base layer, adapted to the characteristics of their device. However they can share the same enhancement layer, since DVC allows encoding frames independently from the reference that will be employed at the decoder.

This approach has been considered in the past in order to improve temporal and spatial scalability. In this work we review the existing approaches, improve them using more recent DVC techniques and perform a new analysis for the emerging multi-view applications.
\end{abstract}

\section{INTRODUCTION}

The Internet is an heterogeneous collection of networks, where users can have different resources in terms of memory and computational complexity. Today the largest part of the Internet traffic is related to video applications such as video conference, video streaming, downloading and sharing. A trivial way to take into account the different requests of the users is to encode the different versions of a video at different qualities and store all the versions on a video server. Then, only one of these versions is sent to each user. Obviously, among the different versions of the same video there will be a huge redundancy. Scalable video coding (SVC) [1] has been developed as an extension of H.264/AVC for encoding the different versions of the video by eliminating redundancies as much as possible. SVC enables to encode the video once, but the users can choose the parameters of the video by selecting only a subset of the bit stream used for encoding the video. Then, the bit stream is divided in a base layer (that consists in the layer at lowest quality) and several enhancement layers, that are sent to the user only if requested. There are three main types of scalability: temporal, spatial and quality. The temporal scalability enables the user to decode the video at lowest frame rate and then progressively enhance the frame rate. This is possible using hierarchical B-frames such as in H.264/AVC. Spatial scalability enables the user to decode the video at different spatial resolutions. Quality scalability means that for each enhancement layer that is sent, the PSNR of the decoded image w.r.t. the base layer one increases. However, besides these "classical" forms of scalability, today new ones appear, associated to the emerging formats such as multi-view video (MVV) [2], [3] and multi-view video-plus-depth (MVD) [4]: we may have view scalability when a subset of the total views is decodable without having to decode all the views, and component scalability when the the access to one component (texture or depth) does not rely on the decoding of the other.

One of drawbacks of classical scalable approaches is that each enhancement layer is strictly dependent from the previous ones. Moreover, an enhanced layer cannot be decoded, if the previous one is not correctly received and decoded. In order to make each layer independent of the others, [5], [6], [7] and [8] propose to apply Distributed Video Coding (DVC) for encoding the video. DVC is based on distributed source coding $[9,10]$. In this paradigm, dependent sources are independently encoded but jointly decoded. Under some constraints on the statistical characteristics of the sources, the loss in terms of rate-distortion performance is negligible w.r.t. classical joint source coding. Concerning scalability, this means that with DVC we can encode the different layers independently. Then, the decoding is independent from which information is available at the decoder side. In this way, we can have different base layers sharing the same enhancement layer encoded in DVC. This can allow remarkable bandwidth savings, above all when many different codecs are considered. Due to the different video coding techniques present nowadays on a network, (for example H.264/AVC with its different profiles, HEVC, MPEG-2, MPEG-4), it would be necessary to encode the enhancement layer of the video in all these formats, if its base layer is in the same format. On the contrary, if DVC is used, one only version of the enhancement layer is sufficient for all the users independently of the technique used for the base layer.

In this paper, we also analyse the RD performance when 
scalable DVC is applied on view domain in the context of multiview distributed video coding. Moreover, several solutions are possible that allow view scalability: of course, a trivial solution is using the same single view encoder on each view (Simulcast); a more effective approach is based on the use of the multiview extension of H.264/AVC, called H.264/MVC. In this work, we compare the performance of multiview scalable DVC w.r.t. these classical approaches for view scalability.

The paper is structured as follows. In Section 2, we provide a state-of-the-art about scalable video coding, distributed video coding and scalable DVC. In Section 3, we describe in detail our analysis and comparison, and we conclude the paper in Section 4.

\section{BACKGROUND IN SCALABILITY AND DVC}

\subsection{Temporal Scalability in H.264/AVC (SVC)}

The scalable extension of H.264/AVC [1] has been proposed in order to take into account the different resources in terms of memory and complexity of the user, for temporal, spatial and quality scalability. Let us consider a video stream divided into a base layer (BL) and in $n$ enhancement layers. The base layer consists of only I frames or P-frames, whose reference frame is in the BL. The $n$ enhancement layers can be obtained by introducing hierarchical B-frames. The B-frames of the $l$-th enhancement layer can be obtained by using as reference the frames of the previous enhancement layers (from 1 to $l-1$ ). With a simple dyadic structure, if the original video is at $f$ frames per second (fps), the BL layer is at $f / N$ fps, where $N=2^{n}$ and the $l$-th enhancement layer will be at $2^{l} \frac{f}{N}$ fps. The H.264/SVC standard also allows a flexible (i.e. nondyadic) definition of temporal dependencies between frames.

\subsection{DVC and DISCOVER interpolation algorithm}

In this section we describe one of the most popular frameworks for DVC, the Stanford codec [11]. In this codec, the video stream is split into Key Frames (KFs) and Wyner-Ziv Frames (WZFs). Borrowing the terminology from the predictive video coding context, a KF and all the following WZFs before the next KF are said to form a group of pictures (GOP). Hence the distance between two successive KFs is called GOP size. The KFs are INTRA coded (i.e. without motion estimation and compensation). The Wyner-Ziv Frames are fed into a systematic channel coder. The systematic part is discarded and the parity bits are sent to the decoder. At the decoder side, an estimation of the Wyner-Ziv Frame is needed. It can be obtained by interpolation of the already decoded frames. This estimation is called Side Information (SI) and it can be considered as a noisy version of the true WZF. The channel decoder must correct these estimation errors by using the parity bits. Then, the encoding of the WZFs is completely independent from how the KFs have been encoded and decoded. The European project DISCOVER [12] implemented the Stanford architecture and defined effective tools for coding the KFs and the WZFs. It has become the reference technique for distributed monoview and multiview video coding. In DISCOVER the SI is generated by a linear motion interpolation algorithm of the closest frames available at the decoder side. In a previous work [13], we have proposed a high order motion interpolation (HOMI) based on 4 images. This algorithm improves the RD performance of classical interpolation techniques.

\subsection{Scalable DVC}

One of the drawbacks of SVC is that each layer depends strictly from the previous ones. With DVC, the different layers can be encoded and decoded independently. This means that the base layer can be encoded with any technique without affecting the decoding of the WZFs. In particular, the temporal scalability is intrinsic in DVC. Indeed, the procedure of encoding and decoding for GOP sizes larger than two is very similar to the structure of hierarchical B-frames of H.264/AVC. Let us consider a GOP size equal to 4 . Then, let $I_{k-2}$ and $I_{k+2}$ be two consecutive KFs. These frames are used for the estimation of the WZF at instant $k$. Once this frame has been decoded, the frame $I_{k}$ is available at the decoder side. It can be used along with the KFs for obtaining the estimation of the WZFs at the instants $k-1$ and $k+1$. Tagliasacchi et al. [8] proposed a temporal scalable DVC for the PRISM codec. In this scheme the base layer has been obtained by H.263+/INTRA. The enhancement layer had been obtained by using algorithms for linear motion interpolation. In [5] and [6] a comparison of temporal scalable DVC w.r.t H.264/AVC has been performed. Moreover, for DVC coding they used an overlapped block motion compensation based side information generation module and an adaptive virtual channel noise model module. They obtained that the RD performance of scalable DVC improves the performance of H.264/INTRA but does not surpass the RD of SVC. Then, they suggest to use DVC only if there are some constraints in terms of complexity and memory at the encoder side. The independence of the enhancement layer w.r.t. the base layer for DVC has been emphasized by [5] and [7]. Indeed, also if we change the anchor frames, the enhancement layers does not change for DVC. On the contrary, another enhancement layer is needed each time that the INTRA Frames of H.264/AVC are coded in a different manner. The quality scalability is also automatically obtained with DVC: the parity bits generated by the encoder are used for improving the quality of the side information. Then, the more parity bits are sent to the decoder, the better the quality of the decoded frames is. Each set of parity bits progressively improves the PSNR of the decoded WZFs. Solutions for spatial scalability have been proposed by [5] and [14]. 
In [15] and [16] the temporal scalability is extended for multiview video coding. Ozbek et al. [15] suppose to have two cameras : the right view is temporally predicted and the left view is predicted from the right one. They extend this structure for multiview by supposing that only one view camera depends from itself and the other ones are predicted by this reference view. Drose et al. [16] suppose that only a central camera is coded independently of the other ones. The temporal stream is coded with a certain GOP structure. In the position of the I frames, the frames of the other cameras are Pframes depending on the frames of the central camera, as the view progressive architecture of H.264/MVC [2]. The other frames are coded only by exploiting temporal correlation.

\section{SYSTEM PERFORMANCE ANALYSIS}

Let us consider now the works of [5] and [6]: we perform here a performance analysis of DISCOVER w.r.t. some relevant video coding standards: H.264/AVC, H.264/AVC with a low complexity profile, the emerging HEVC. The low-complexity profile of H.264/AVC is obtained by switching off the rate distortion optimization.

In our use-case, we have to send the different bit streams of the different standards. If a user having the BL of H.264/AVC cannot decode the B-frames encoded with HEVC and viceversa. For these reasons, it is necessary to send the EL bitstreams of H.264/AVC and HEVC. But if we suppose that all the users have a DVC decoder, the enhancement layers can be coded with a Wyner-Ziv codec, and thus one bitstream is sufficient for all the users. We have extended the temporal scalable video coding along the view axis in multiview video. We suppose that we have $K$ cameras. One camera out of $V$ is a Key camera. The other ones are Wyner-Ziv cameras. The base layer consists of sending only the Key views. The other views are hierarchical encoded, as in the temporal domain, as depicted in Fig. 1. Let us suppose that one out of four cameras is a Key camera and let 0 and 4 be two of these cameras. Then, in the first enhancement layer, the view number 2 is sent and for the second layer the cameras 1 and 3 are sent. This structure is used both for the DVC codec and for H.264/MVC. If the DVC DISCOVER is not used, in order to take into account that some users can not have the H.264/MVC decoder, we are forced to encode and store also a simulcast version of this video, where each camera is independently coded. For this reasons, the performance of scalable multiview distributed video coding are compared w.r.t. H.264/MVC and H.264/Simulcast.

In order to perform a complete analysis of the different methods for scalable video coding, we suppose that we have two scenarios. In the first scenario, the users have different decoders: we suppose that each video stored on the video server is coded in H.264/AVC, H.264/AVC low-complexity (with no RD optimization) and HEVC. Even if the base layers 1 and 2 are compatible, the corresponding enhancement lay-

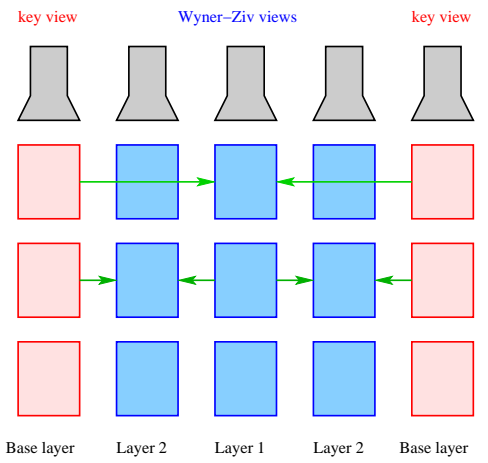

Fig. 1. Example of multiview SVC with $V=4$

ers will not be, since they are predicted w.r.t. possibly different images. In this context we are then obliged to send all the base layers and all the enhancement layers. Another scenario is that only the base layer is INTRA coded with H.264/AVC or HEVC, and the enhancement layers are encoded with the DISCOVER DVC scheme. These means that the enhancement layers are independent from the base layer available for each user. For the scalable monoview we have considered the MPEG sequences party scene and BQSquare, respectively at spatial resolutions of $832 \times 480$ and $416 \times 240$. Their frame rates are of $60 \mathrm{fps}$ and $50 \mathrm{fps}$ respectively. We have considered a GOP size of 4, and then we can suppose that we have a base layer and two enhancement layers. The frame rate for the base layer is respectively $12.5 \mathrm{fps}$ and $15 \mathrm{fps}$. We have then considered DISCOVER with the base layer (that means the KFs) encoded with H.264/AVC, with HEVC and H.264/AVC (low complexity). We have performed a rate-distortion analysis of DVC w.r.t. the scenario where we are obliged to send H.264/AVC, HEVC and H.264/AVC low complexity (see Tab. 1) and we have obtained up to $23.58 \%$ of bit reduction and up to $3.54 \mathrm{~dB}$ of PSNR improvement. Indeed, when standard video techniques are used for the enhancement layers, we are obliged to encoded these layers with all the considered standard. With DVC, since the enhancement layers are independent of the BL, we can use the same set of parity bits independently of which BL is available to the user.

In the context of multiview video coding, we have considered the Xmas sequence at $480 \times 640$ spatial resolution and we have compared the RD performance of DVC w.r.t. Simulcast+H.264/MVC (see Fig. 2 and 3). Indeed, if some users have not the H.264/MVC codec, we are forced to send on the net also the Simulcast version, where all the views are independently encoded.

\section{CONCLUSIONS AND FUTURE WORKS}

In this paper we have performed an analysis in terms of RD performance for temporal scalable DVC w.r.t. classical scal- 


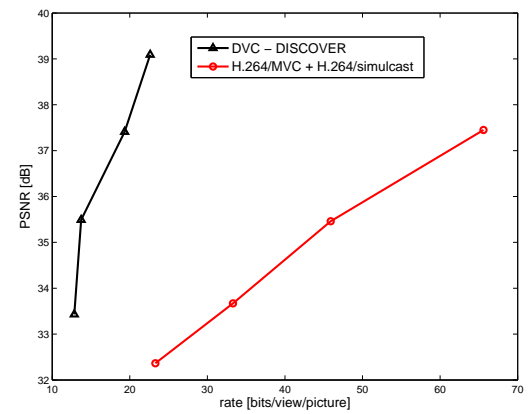

Fig. 2. RD curves for sequence Xmas - Layer 1

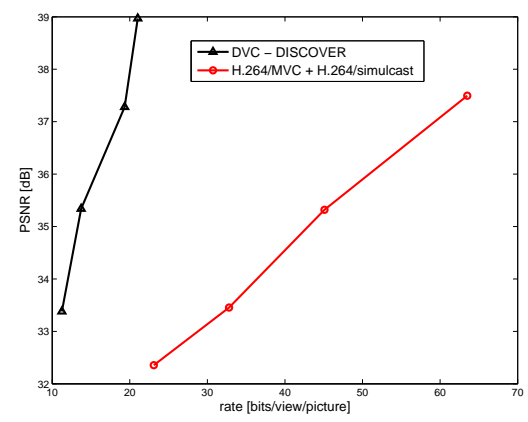

Fig. 3. RD curves for sequence Xmas - Layer 2

able techniques. In contrast with the classical case, enhancement layers in DVC are independent from the BL. Then, if different users have different decoders, using DVC, the same parity bits will be sufficient to decode the enhancement layers independently of the codec used for the base layer, thus achieving a noticeable bandwidth saving. We have extended our analysis also to multiview video coding, in order to take into account that some users can have the H.264/MVC codec and others may not have it. Then, we should send also the Simulcast version of this video. If DVC is used, we can avoid to send these two versions.

\section{REFERENCES}

[1] H. Schwarz, D. Marpe, and T. Wiegand, "Overview of the scalable video coding extension of the H.264/AVC standard," IEEE Transactions on Circuits and Systems for Video Technology, vol. 17, no. 9, pp. 1103-1120, Sept. 2007.

[2] A. Vetro, T. Wiegand, and G.J. Sullivan, "Overview of the stereo and multiview video coding extensions of the h.264/mpeg-4 avc standard," Proceedings of the IEEE, vol. 99, no. 4, pp. 626 -642, april 2011.

[3] Ying Chen, Ye-Kui Wang, Kemal Ugur, Miska M. Hannuksela, Jani Lainema, and Moncef Gabbouj, "The emerging MVC standard for 3D video services," EURASIP J. Appl. Signal Process., vol. 2009, pp. 8:18:13, January 2008.

[4] P. Merkle, A. Smolic, K. Muller, and T. Wiegand, "Multi-view video plus depth representation and coding," in Proceedings of IEEE Internantional Conference on Image Processing, San Antonio, TX, 2007.

\begin{tabular}{|l|c|c|}
\hline method & $\Delta_{R}[\%]$ & $\Delta_{P S N R}[\mathrm{~dB}]$ \\
\hline \multicolumn{3}{|c|}{ BQSquare - layer 1 } \\
DVC (KF coded with H.264/AVC ) & -4.70 & 0.86 \\
DVC (KF coded with HEVC) & -23.58 & 0.40 \\
DVC (KF coded with H.264/AVC 1.c.) & -4.73 & 0.20 \\
\hline \multicolumn{3}{|c|}{ BQSquare - layer 2 } \\
DVC (KF coded with H.264/AVC ) & 19.17 & 3.54 \\
DVC (KF coded with HEVC) & 4.24 & 0.83 \\
DVC (KF coded with H.264/AVC 1.c. ) & 20.20 & 0.84 \\
\hline \multicolumn{2}{|c|}{ Party Scene - layer 1 } \\
DVC (KF coded with H.264/AVC ) & -12.79 & 0.80 \\
DVC (KF coded with HEVC) & -16.36 & 1.07 \\
DVC (KF coded with H.264/AVC 1.c.) & -11.56 & 0.78 \\
\hline \multicolumn{2}{|c|}{ Party Scene - layer 2 } \\
DVC (KF coded with H.264/AVC ) & -13.71 & 1.06 \\
DVC (KF coded with HEVC ) & -18.22 & 1.08 \\
DVC (KF coded with H.264/AVC 1.c. ) & -12.89 & 1.02 \\
\hline
\end{tabular}

Table 1. RD performance by Bjontegaard metric w.r.t. H.264+HEVC+H.264(low-complexity)

[5] M. Ouaret, F. Dufaux, and T. Ebrahimi, "Codec-independent scalable distributed video coding," in Image Processing, 2007. ICIP 2007. IEEE International Conference on, 16 2007-oct. 19 2007, vol. 3, pp. III -9 $-\mathrm{III}-12$.

[6] X. Huang, A. Ukhanova, E. Belyaev, and S. Forchhammer, "Temporal scalability comparison of the h. 264/svc and distributed video codec," in Ultra Modern Telecommunications \& Workshops, 2009. ICUMT'09. International Conference on. IEEE, 2009, pp. 1-6.

[7] M. Ouaret, F. Dufaux, and T. Ebrahimi, "Error-resilient scalable compression based on distributed video coding," Signal Processing: Image Communication, vol. 24, no. 6, pp. 437-451, 2009.

[8] M. Tagliasacchi, A. Majumdar, and K. Ramchandran, "A distributedsource-coding based robust spatio-temporal scalable video codec," in Proc. Picture Coding Symposium, 2004.

[9] D. Slepian and J. K. Wolf, "Noiseless coding of correlated information sources," IEEE Transactions on Information Theory, vol. 19, pp. 471480, July 1973.

[10] A. Wyner and J. Ziv, "The rate-distortion function for source coding with side information at the receiver," IEEE Transactions on Information Theory, vol. 22, pp. 1-11, Jan. 1976.

[11] A. Aaron, R. Zhang, and B. Girod, "Wyner-Ziv coding of motion video," in Asilomar Conference on Signals and Systems, Pacific Grove, California, Nov. 2002.

[12] X. Artigas, J. Ascenso, M. Dalai, S. Klomp, D. Kubasov, and M. Ouaret, "The Discover codec: Architecture, techniques and evaluation," in Proceedings of Picture Coding Symposium, Lisbon, Portugal, Nov. 2007.

[13] G. Petrazzuoli, M. Cagnazzo, and B. Pesquet-Popescu, "High order motion interpolation for side information improvement in DVC," in Proceedings of IEEE International Conference on Acoustics, Speech and Signal Processing, Dallas, TX, 2010.

[14] B. Macchiavello, F. Brandi, R.L. Queiroz, and D. Mukherjee, "Superresolution applied to distributed video coding with spatial scalability," Anais do Simpsio Brasileiro de Telecomunicaes, 2008.

[15] N. Ozbek and A. Tekalp, "Scalable multi-view video coding for interactive 3dtv," in Multimedia and Expo, 2006 IEEE International Conference on. IEEE, 2006, pp. 213-216.

[16] M. Drose, C. Clemens, and T. Sikora, "Extending single-view scalable video coding to multi-view based on h. 264/avc," in Image Processing, 2006 IEEE International Conference on. IEEE, 2006, pp. 2977-2980. 